\title{
Characterizing Software Developers by Perceptions of Productivity
}

\author{
Meyer, André N ; Zimmermann, Thomas ; Fritz, Thomas
}

\begin{abstract}
Understanding developer productivity is important to deliver software on time and at reasonable cost. Yet, there are numerous definitions of productivity and, as previous research found, productivity means different things to different developers. In this paper, we analyze the variation in productivity perceptions based on an online survey with 413 professional software developers at Microsoft. Through a cluster analysis, we identify and describe six groups of developers with similar perceptions of productivity: social, lone, focused, balanced, leading, and goal-oriented developers. We argue why personalized recommendations for improving software developers' work is important and discuss design implications of these clusters for tools to support developers' productivity.
\end{abstract}

DOI: https://doi.org/10.1109/ESEM.2017.17

Posted at the Zurich Open Repository and Archive, University of Zurich ZORA URL: https://doi.org/10.5167/uzh-141848

Conference or Workshop Item

Originally published at:

Meyer, André N; Zimmermann, Thomas; Fritz, Thomas (2017). Characterizing Software Developers by Perceptions of Productivity. In: International Symposium on Empirical Software Engineering and Measurement, Toronto, 9 November 2017 - 10 November 2017.

DOI: https://doi.org/10.1109/ESEM.2017.17 


\title{
Characterizing Software Developers by Perceptions of Productivity
}

\author{
André N. Meyer ${ }^{1}$, Thomas Zimmermann ${ }^{2}$, Thomas Fritz ${ }^{1,3}$ \\ ${ }^{1}$ University of Zurich, Zurich, Switzerland, \{ameyer, fritz $\} @$ ifi.uzh.ch \\ ${ }^{2}$ Microsoft Research, Redmond, WA, USA, tzimmer@microsoft.com \\ ${ }^{3}$ University of British Columbia, Vancouver, BC, Canada, fritz@cs.ubc.ch
}

\begin{abstract}
Understanding developer productivity is important to deliver software on time and at reasonable cost. Yet, there are numerous definitions of productivity and, as previous research found, productivity means different things to different developers. In this paper, we analyze the variation in productivity perceptions based on an online survey with $\mathbf{4 1 3}$ professional software developers at Microsoft. Through a cluster analysis, we identify and describe six groups of developers with similar perceptions of productivity: social, lone, focused, balanced, leading, and goal-oriented developers. We argue why personalized recommendations for improving software developers' work is important and discuss design implications of these clusters for tools to support developers' productivity.
\end{abstract}

Keywords - productivity, perceptions, software developers

\section{INTRODUCTION}

Understanding, measuring and optimizing software developers' productivity is important to deliver software on time and at reasonable cost and quality. Previous work introduced numerous measures of productivity that vary by their focus on specific outputs of development work, such as lines of code [1][2], function points [3], or completed tasks [4], over time. Other researchers have looked at organizational factors and their impact on developer productivity, such as the team [5]-[7] and workplace characteristics [8]. While these measures and factors can be valuable to compare certain aspects of productivity, they neglect to capture the many differences in perceptions of productivity as well as the differences in developers' work, roles and habits. By investigating developers' perceptions of productivity, several researchers concluded that developers are different in what they consider as productive or unproductive [6][9]-[11]. Yet, little is known about the characteristics, the variation, and the commonalities in developers' productivity perceptions. A better understanding of these aspects of developers' productivity perceptions can help to provide better and more tailored support to developers.

In this paper, we explore the characteristics of developers' perceptions of productivity and the clusters of developers with similar perceptions. We report on the results from an online survey with 413 professional software developers at Microsoft. We show that developers can roughly be clustered into six groups with similar perceptions - social, lone, focused, balanced, leading, and goal-oriented developer - thus allowing to abstract and simplify the variety of individual productivity perceptions. We characterize these groups based on the aspects that developers perceive to influence their productivity positively or negatively, and by the measures developers are interested in to reflect about their productivity. We discuss the implications of our clusters on software development and their potential in optimizing developers' productivity and support tools.

\section{RELATED WORK}

Since the 1970s researchers have been investigating how various factors influence developer productivity. Following Wagner's and Ruhe's categorization [12], these factors include, amongst others, technical factors, such as the programming language [13], software tools [14], software size and complexity [5][15] and product quality [8]; and social factors, such as team and turnover [5]-[7], experience and skills [5][14], and workplace environment [8]. Recently, an increasing amount of research has focused on the influence of different work habits and patterns on productivity. For instance, a high fragmentation of work and multi-tasking have been shown to decrease productivity due to long resumption lags and difficulties in switching between work contexts [10][16]-[18]. Similarly, meetings-another source of fragmentation - can have a negative impact on productivity, especially when they have no clear goal or are not well prepared [9]. Breaks that refresh and relax a person can, however, have a positive effect on productivity despite their impact on work fragmentation [19]. These and other factors and patterns likely affect most developers in some way, yet the impact on their productivity differs greatly and is highly individual due to differences, such as developers' experiences, projects, job titles, and work set-ups [6][9]. In our work, we make a first attempt at grouping developers with similar perceptions together based on the perceived impact of these factors on their productivity.

\section{Methodology}

To explore developers' productivity perceptions, in particular the variations and similarities amongst developers, we designed and conducted a survey and analyzed the collected answers from 413 participants. 


\section{A. Data Collection}

We conducted an online survey, consisting of four main questions about productivity perceptions, at Microsoft.

Survey Design. The first two questions Q1 and Q2 asked the participants to describe a productive and an unproductive work day in two words each ("Please describe what a productive work day is to you in two words.", "Please describe what an unproductive work day is to you in two words."). We prompted users for two keywords each to foster more than one precise definition of productivity, similar to what we applied in a previous study [9]. The third question $\mathbf{Q 3}$ asked the agreement with statements on factors that might affect productivity. The last question Q4 asked about the interestingness of productivity measures at work. The order of the questions was chosen to not bias the participants when they described productive and unproductive work days (Q1 and Q2), before showing them the list of statements and measures (Q3 and Q4). The complete survey can be found as supplementary material ${ }^{1}$. None of the questions were required to be answered and participants could stop the survey at any point in time.

Productivity perceptions (Q3). For question Q3, "Please rate your agreement with each of the following statements", we used a symmetric, five-point Likert scale from strongly agree (5) to strongly disagree (1) to ask about the agreement with 20 statements on when people feel productive, for example, "I feel productive when I write code." The statements were selected from related work that analyzed the impact of various work patterns on productivity. We focused on statements about activities software developers pursue during a work day and the fragmentation of their work. Specifically, we asked participants about the perceived relation between productivity and coding related activities (that is, their main work activity) [9][13]; social activities such as emails [20], meetings [9], and helping co-workers [9][21]; work unrelated activities such as breaks [19]; the fragmentation of their work such as the impact of distractions and multi-tasking [8][16][17]; the time of the day [22]-[24]; and their happiness at work [25][26].

Productivity measures (Q4). For question Q4, "Please rate how interesting each of the following items would be for you to reflect on your work day or work week", we used a symmetric, five-point Likert scale from extremely interesting (5) to not at all interesting (1) to ask about the interestingness of 30 potential measures of productivity to reflect about work, such as "The time I spent coding." or "The number of emails I sent." The measures were selected as follows: for the categories, which we identified for Q3 from related work, we selected measures related to how much time was spent on an activity/event and the total number of times an activity/event occurred. We further added measures related to the overall time spent on the computer and within various applications, and the tasks worked on, which developers in another study on productivity considered to be most relevant [9].
Participants. We advertised the survey by sending personalized invitation emails to 1600 professional software developers within Microsoft. To incentivize participation, we held a raffle for two US\$ 50 gift certificates. In total, 413 people participated in the survey (response rate of $25.8 \%$ ); $91.5 \%$ of the participants reported their role to be individual contributor, $6.8 \%$ team lead or manager, and $1.7 \%$ stated they are neither. Participants had an average of 9.6 years ( \pm 7.5 , ranging from 0.3 to 36 ) of professional software development experience.

\section{B. Data Analysis}

We used the responses to Q3 to group participants with similar perceptions of productivity together.

First, we normalized the responses. When responding to surveys, some participants are more positive than others, which can lead to biases in the responses. For example, Alice might center her responses to the question Q3 around the response to "agree", while Bob tends to center his responses around the response "neutral". To correct for such personal tendencies, we normalized responses to Q3 and Q4 as follows. We treated the scale as numeric and for each survey participant we computed the median response for Q3 and Q4 respectively: median $Q 3$ and medi$a n_{Q 4}$. We then subtracted the median from the responses and computed the sign. More formally, for the response $r_{q, I}$ to a question $q$ and item $I$, we normalize with $\operatorname{sign}\left(r_{q, I}-\right.$ median $\left._{q}\right)$. As a result, we end up with three categories: A value of +1 indicates that a participant responded more positively about an item than for most of the other items (HIGHER). A value of -1 indicates that a participant was more negative about an item (LOWER). A value of 0 indicates that a participant was neutral towards an item. We used medians instead of means because they more effectively capture neutral responses as zero.

Next, we clustered participants into groups using the pamk function from the $f p c$ package in R. The input was the normalized responses to Q3. The pamk function is a wrapper function to the commonly used pam clustering function. The wrapper computes the optimal number of clusters. In our case, the optimal number of clusters was six. The resulting clusters - social, lone, focused, balanced, leading, and goal-oriented developers - are discussed in the next section.

Finally, to describe the inferred groups, we used the responses to questions Q1, Q2, and Q3. We created comparison word clouds for the responses to Q1 and Q2 using the wordcloud package in $\mathrm{R}$. These word clouds depict the relative frequency of the most frequently used words for each cluster, with more frequently used words being displayed in a bigger font size. Furthermore, we used the responses to Q4 to identify the measures that developers of a cluster are interested in. 


\section{Cluster 2}

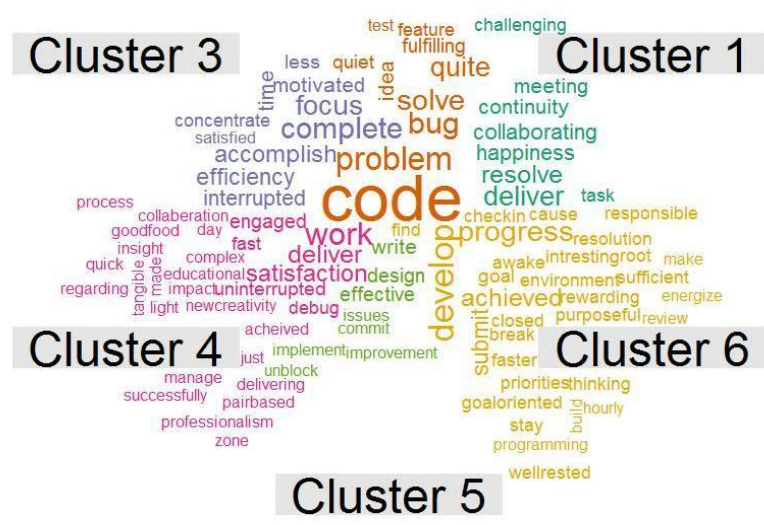

Cluster 2

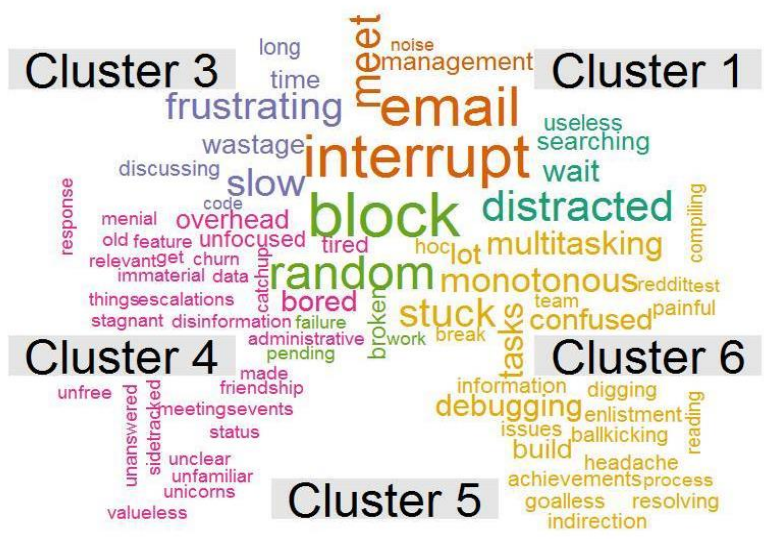

Fig. 1: Comparing the clusters with respect to words that developers associate with productive (left, Q1) and unproductive work days (right, Q2). Terms in turquoise are related to Cluster 1, orange to Cluster 2, purple to Cluster 3, pink to Cluster 4, green to Cluster 5, and gold to Cluster 6. The size of a term corresponds to the difference between the maximum relative frequency and the average relative frequency of the word across the six clusters.

\section{RESULTS}

We identified the following six clusters based on our analysis of Q3. We further describe them based on the responses from Q1, Q2 (see the word clouds in Figure 1, one color per cluster) and Q4:

1. The social developers (C1) feel productive when helping coworkers, collaborating and doing code reviews. To get things done, they come early to work or work late and try to focus on a single task.

2. The lone developers (C2) avoid disruptions such as noise, email, meetings, and code reviews. They feel most productive when they have little to no social interactions and when they can work on solving problems, fixing bugs or coding features in quiet and without interruptions. To reflect about work, they are mostly interested in knowing the frequency and duration of interruptions they encountered.

3. The focused developers (C3) feel most productive when they are working efficiently and concentrated on a single task at a time. They are feeling unproductive when they are wasting time and spend too much time on a task, because they are stuck or working slowly. They are interested in knowing the number of interruptions and focused time.

4. The balanced developers (C4) are less affected by disruptions. They are less likely to come early to work or work late. They are feeling unproductive, when tasks are unclear or irrelevant, they are unfamiliar with a task, or when tasks are causing overhead.

5. The leading developers (C5) are more comfortable with meetings and emails and feel less productive with coding activities than other developers. They feel more productive in the afternoon and when they can write and design things. They don't like broken builds and blocking tasks, preventing them (or the team) from doing productive work.
6. The goal-oriented developers (C6) feel productive when they complete or make progress on tasks. They feel less productive when they multi-task, are goal-less or are stuck. They are more open to meetings and emails compared to the other clusters, in case they help them achieve their goals.

Table 1 describes the characteristics of the clusters in more detail, in particular the cluster name, the statements for which half or more participants in the cluster gave HIGHER scores for Q3 (second column), and the statements for which half or more participants in the cluster gave LOWER scores for Q3 (third column). Prefixed with $\widehat{\Upsilon}$, the table also lists the productivity measures (from question Q4) which were interesting (second column) or not interesting (third column) to the majority of developers within a cluster. The tendency reported in the table corresponds to the average normalized score. If the tendency is not reported for a statement, it means it was greater than 0.500 (second column) or lower than -0.500 (third column).

The first row lists the statements that were scored higher/ lower by most participants (50\% or more) in the majority of clusters (four or more). As an example, the statement "I feel productive when I write code" was scored higher by more than $50 \%$ of people in clusters $\mathrm{C} 1, \mathrm{C} 2, \mathrm{C} 3, \mathrm{C} 4$, and C6. This was not the case for cluster $\mathrm{C} 5$, which is reported as an exception, both in the first row and the row corresponding to C5. Other statements scored higher by most developers in most clusters are "I feel productive on a day with little to no meetings", "I feel productive when I am happy", and "I feel productive when I have fewer interruptions".

Table I also shows that some measurements $(\boldsymbol{})$ are of interest for reflection on work (Q4) to most clusters. People in most clusters gave higher scores to the time spent coding and the longest period focused on a task without an interruption. The number of open applications and the inbox size received lower scores overall. However, the table also highlights differences between 
the clusters with respect to the measurements that participants consider to be interesting. For example, the lone developers $(\mathrm{C} 2)$ are interested in the number and duration of interruptions. They are less interested in the list of applications used and web sites visited. The balanced developers (C4) are interested in the tasks, the number of interruptions, and the focus over time. They are less interested in the number of emails sent and received.

Several clusters are further related to each other along specific aspects. For example, $\mathrm{C} 1$ and $\mathrm{C} 2$ are related in how they perceive the productivity of social interactions. While social developers $(\mathrm{C} 1)$ embrace them, lone developers $(\mathrm{C} 2)$ feel more productive when having uninterrupted work alone. Further, clusters $\mathrm{C} 3$ and $\mathrm{C} 6$ are related, as focused developers (C3) are more interested about working efficiently, while goal-oriented developers (C6) feel the most productive when they get work done.

\section{DISCUSSION}

Understanding how developers perceive productivity is important to better support them and foster productivity. The six clusters and their characteristics provide relevant insights into groups of developers with similar productivity perceptions that can be used to optimize the work and flow on the team and the individual level. The differences between software developers' preferred collaboration and work styles show that not all developers are alike, and that the cluster an individual or team belongs to could be a basis for tailoring actions for improving their work and productivity.

On the team level, it might, for instance, be most beneficial to provide a quiet, less interruption-prone office space to lone and focused developers ( $\mathrm{C} 2$ and $\mathrm{C} 3$ ) and seat social developers (C1) in open offices. Similarly, a team might benefit from an increased awareness about each members' communication preferences, to reduce ad-hoc meetings for lone and focused developers ( $\mathrm{C} 2$ and $\mathrm{C} 3$ ) or use more asynchronous communication where they can choose when to respond to an inquiry. The group of developers can be further beneficial for task assignment. For example, an exploration task for a new product that is rather open without clear goals and that requires a lot of discussion might be less suitable for a goal-oriented $(\mathrm{C} 6)$, a lone $(\mathrm{C} 2)$ or a balanced developer (C4).

On the individual level, developers might benefit from tailored user experiences and feature sets for software development tools. For instance, a tool to foster productive work and avoid interruptions could block emails and instant messaging notifications for the lone developer $(\mathrm{C} 2)$ while they are coding, but allow them for the social developer $(\mathrm{C} 1)$, similar to what was previously suggested [27]. Similarly, the code review or build experience could be adjusted based on different productivity perceptions. In addition, the clusters could be used for advice tailored to specific groups of developers, e.g., recommend the focused developer (C3) to come to work early to have uninterrupted work time, or suggest the balanced developer (C4) to take a break to avoid boredom and tiredness [19]. The clusters can help to quantify the individual productivity of developers more accurately, by considering what matters most to them and depending on their perceptions of productivity. For example, a leading developer (C5) is likely feeling much more productive after a day with multiple meetings spread over the day, compared to the focused developer (C3), who only has little time to focus on the tasks in-between these meetings.

Overall, the identified clusters and the aspects that differentiate these clusters, such as goal-orientation, single-task focus or socialness, are a first step towards a set of "productivity traits" of developers. Similar to the big five personality traits (OCEAN) [28] that help to understand other people's personality, the selfassessment along such productivity traits can provide useful information for understanding oneself or other developers and for optimizing the work individually as well as in teams.

\section{THREATS TO VALIDITY}

We briefly discuss threats to validity.

External validity. Due to the selection of participants, as all work for the same company, the results might not generalize to other software development contexts. We tried to mitigate this threat by advertising the survey to professional software developers in different product teams within Microsoft, at different stages in their projects, and with varying amounts of experience; resulting in a more diverse set of participants. By providing the survey questions, we encourage other researchers and practitioners to replicate the study in other companies.

Construct validity. The selection of questions that we asked in the survey also impacts the results. For example, questions about different dimensions of productivity, might lead to a different clustering. We created the questions based on factors that we identified in related work and from our previous experience with surveying and interviewing developers about their perceptions and measuring of productivity [9]. The choice of clustering algorithm and approach of using questions Q1 to Q3 to describe the inferred clusters might also have influenced the results. Future work is needed to analyze the robustness and completeness of the productivity statements and clusters.

\section{CONCLUSION}

Different to previous work that suggested numerous productivity measures and found that perceptions of productivity can vary greatly between developers, our research provides an exploratory first step into identifying commonalities and underlying categories of developers' productivity perceptions. Based on the clustering of developers' answers to productivity statements mentioned in related work, we identified and characterized an initial set of six such categories and discussed their potential to improve the work and flow of software developers and their teams.

\section{ACKNOWLEDGEMENTS}

The authors would like to thank the study participants. 


50\% OR MORE GIVE HIGHER SCORES FOR
(TENDENCY: > 0.500)

Cluster C4:

The balanced

developer

Size: 50 developers

Cluster C5: $\quad$ Exception: I feel productive when I write code (tendency:

$0.309) \bullet$ Exception: I feel productive on a day with little to no meetings (tendency: -0.103$) \bullet$ Exception: I feel productive when I am happy (tendency: 0.268 )
The leading developer

Size: 97 developers interruptions (tendency: 0.247)

\begin{abstract}
Exception: I feel productive when I have fewer
\end{abstract}
$50 \%$ OR MORE GIVE LOWER SCORES FOR (TENDENCY: $<-0.500)$
I feel productive when I send more emails than usual (except C6) $\bullet$ I feel I had a productive work day when my email inbox is emptier in the evening than in the morning (except $\mathrm{C} 1$ and $\mathrm{C} 4) \bullet$ I feel productive when I visit social networks or news websites to do a quick break (except $\mathrm{C} 1$ and $\mathrm{C} 4$ ) $\bullet$ If I have many program windows open on my screen, it decreases my perceived productivity $\bullet$ I feel productive on a particular day of the week, e.g., on Wednesdays (except C5) $\bullet$ I feel more productive in the morning than in the afternoon (except $\mathrm{C} 3$ ) $\bullet$ I feel less productive after lunch compared to the rest of the day (except $\mathrm{C} 3$ and C6)

$\checkmark$ The number of open application windows (except $\mathrm{C} 1$ and $\mathrm{C5}$ )

$\checkmark$ The inbox size in the course of the day/week (except C2, C5)

Exception: I feel I had a productive work day when my email inbox is emptier in the evening than in the morning (tendency: -0.258)

Exception: I feel productive when I visit social networks or news websites to do a quick break (tendency: -0.403 )

$\checkmark$ Exception: The number of open application windows (tendency: -0.370 )
I feel productive when I do code reviews

$\checkmark$ A list of applications I used

$\checkmark$ The websites I visited the most

$\checkmark$ Exception: The inbox size in the course of the day/week (tendency: -0.438)
I feel more productive in the afternoon than in the morning • Exception: I feel less productive after lunch compared to the rest of the day (tendency -0.155 )

$\checkmark$ The number of emails I received

$\checkmark$ The number of emails I sent
I come early to work/work late to get some focused work hours $\bullet$ Exception: I feel I had a productive work day when my email inbox is emptier in the evening than in the morning (tendency: -0.180) • Exception: I feel productive when I visit social networks or news websites to do a quick break (tendency 0.000 )
I feel more productive in the afternoon than in the morning $\bullet$ Exception: I feel productive on a particular day of the week, e.g., on Wednesdays (tendency: -0.400 )

$\checkmark$ Exception: The number of open application windows (tendency: -0.447)

$\checkmark$ Exception: The inbox size in the course of the day/week (tendency: -0.478)
Exception: I feel productive when I send more emails than usual (tendency 0.135) • Exception: I feel less productive after lunch compared to the rest of the day (tendency: -0.211 )

\section{The goal-oriented} developer

Size: 38 developers
I feel productive when I work on one task at a time $\bullet$ Exception: I feel productive on a day with little to no meetings (tendency -0.079) • Exception: I feel productive when I have fewer interruptions (tendency: 0.447) 


\section{REFERENCES}

[1] P. Devanbu, S. Karstu, W. Melo, and W. Thomas, "Analytical and empirical evaluation of software reuse metrics," in Software Engineering, 1996., Proceedings of the 18th International Conference on, 1996, no. August, pp. 189-199.

[2] C. E. Walston and C. P. Felix, "A Method of Programming Measurement and Estimation,” IBM Syst. J., vol. 16, pp. 54--73, 1977.

[3] U. Conf, M. Kaufman, S. Mateo, and C. Jones, "Software metrics: good, bad and missing," Computer (Long. Beach. Calif)., vol. 27, no. 9, pp. 98-100, 1994.

[4] M. Zhou and A. Mockus, "Developer fluency: Achieving true mastery in software projects.," in Proceedings of the eighteenth ACM SIGSOFT international symposium on Foundations of software engineering - FSE '10, 2010, p. 137.

[5] B. W. Boehm et al., Software Cost Estimation with Cocomo II with Cdrom. Prentice Hall PTR, 2000.

[6] C. Melo, D. S. Cruzes, F. Kon, and R. Conradi, "Agile team perceptions of productivity factors," Proc. - 2011 Agil. Conf. Agil. 2011, pp. 57-66, 2011.

[7] J. D. Blackburn, G. D. Scudder, and L. N. Van Wassenhove, "Improving speed and productivity of software development: a global survey of software developers," IEEE Trans. Softw. Eng., vol. 22, no. 12, pp. 875-885, 1996.

[8] T. DeMarco and T. Lister, Peopleware: Productive Projects and Teams. Addison-Wesley Professional, 2013.

[9] A. N. Meyer, T. Fritz, G. C. Murphy, and T. Zimmermann, "Software Developers' Perceptions of Productivity," in Proceedings of the 22Nd ACM SIGSOFT International Symposium on Foundations of Software Engineering, 2014, pp. 19-29.

[10] B. Vasilescu et al., "The Sky is Not the Limit: Multitasking on GitHub Projects,” 2016, pp. 994-1005.

[11] P. M. Johnson, J. Agustin, C. Chan, C. Moore, J. Miglani, and W. E. J. Doane, "Beyond the Personal Software Process: Metrics collection and analysis for the differently disciplined," 25th Int. Conf. Softw. Eng. 2003. Proceedings., vol. 6, pp. 641-646, 2003.

[12] S. Wagner and M. Ruhe, "A Systematic Review of Productivity Factors in Software Development," in Software Productivity Analysis and Cost Estimation (SPACE 2008), 2008, pp. 1-6.

[13] A. J. Albrecht, "Measuring application development productivity," IBO Conference on Application Development. pp. 83-92, 1979.

[14] P. D. Chatzoglou and L. A. Macaulay, "The importance of human factors in planning the requirements capture stage of a project," Int. J. Proj. Manag., vol. 15, no. 1, pp. 39-53, Feb. 1997.

[15] F. P. Brooks Jr., The mythical man-month (anniversary ed.). Boston,
MA, USA: Addison-Wesley Longman Publishing Co., Inc., 1995.

[16] V. M. González and G. Mark, “'Constant, Constant ,Multi-tasking Craziness': Managing Multiple Working Spheres,” vol. 6, no. 1, pp. 113-120, 2004.

[17] G. Mark, S. T. Iqbal, M. Czerwinski, P. Johns, and A. Sano, "Neurotics Can't Focus: An in situ Study of Online Multitasking in the Workplace," in Proceedings of the SIGCHI Conference on Human Factors in Computing Systems, 2016, pp. 1739-1744.

[18] A. N. Meyer, L. E. Barton, G. C. Murphy, T. Zimmermann, and T. Fritz, "The Work Life of Developers: Activities, Switches and Perceived Productivity," IEEE Trans. Softw. Eng., vol. PP, no. 99, pp. $1-15,2017$.

[19] D. A. Epstein, D. Avrahami, and J. T. Biehl, "Taking 5: Work-Breaks, Productivity, and Opportunities for Personal Informatics for Knowledge Workers," in Proceedings of the 2016 CHI Conference on Human Factors in Computing Systems - CHI '16, 2016.

[20] G. Mark, S. T. Iqbal, M. Czerwinski, P. Johns, and A. Sano, "Email Duration, Batching and Self-interruption: Patterns of Email Use on Productivity and Stress," in Proceedings of the 2016 CHI Conference on Human Factors in Computing Systems - CHI '16, 2016, vol. 21, no. 1, pp. 98-109.

[21] J. Chong and R. Siino, "Interruptions on Software Teams : A Comparison of Paired and Solo Programmers," pp. 29-38, 2006.

[22] J. B. Spira and J. B. Feintuch, "The Cost of Not Paying Attention: How Interruptions Impact Knowledge Worker Productivity,” 2005.

[23] R. Sach, H. Sharp, and M. Petre, "What makes software engineers go that extra mile?," in 23rd Annual Psychology of Programming Interest Group 2011, 2011.

[24] G. Mark, S. T. Iqbal, M. Czerwinski, and P. Johns, "Bored Mondays and Focused Afternoons: The Rhythm of Attention and Online Activity in the Workplace," 2014.

[25] D. Graziotin, X. Wang, and P. Abrahamsson, "Happy software developers solve problems better: psychological measurements in empirical software engineering.," PeerJ, vol. 2, p. e289, Jan. 2014.

[26] I. A. Khan, W.-P. Brinkman, and R. M. Hierons, "Do moods affect programmers' debug performance?," Cogn. Technol. Work, vol. 13, no. 4, pp. 245-258, Oct. 2010.

[27] E. Agapie, D. Avrahami, and J. Marlow, "Staying the Course : SystemDriven Lapse Management for Supporting Behavior Change," in Proceedings of the 2016 CHI Conference on Human Factors in Computing Systems, 2016, p. Proceedings of the 2016 CHI Conference on Human $\mathrm{Fa}$.

[28] S. John, Oliver P and Srivastava, "The Big Five trait taxonomy: History, measurement, and theoretical perspectives," Handb. Personal. Theory Res., vol. 2, pp. 102--138, 1999. 